\title{
The Development of a Fine- and Microstructure Profiler*
}

\author{
RAYMOND W. SCHMITT, JOHN M. TOOLE, RICHARD L. KOEHLER, \\ EDWARD C. MELlinger AND KENNETH W. DOHERTY \\ Woods Hole Oceanographic Institution, Woods Hole, Massachusetts
}

(Manuscript received 3 July 1987, in final form 10 December 1987)

\begin{abstract}
A new freely-falling ocean profiler for measuring finescale variations in temperature, salinity and horizontal velocity and microscale variations in temperature, conductivity, velocity and refractive index has been constructed. The High Resolution Profiler (HRP) features: full ocean depth capability, a microcomputer to control sampling, data storage and operations, solid state mass storage, and commercially available sensors. A special handling rig facilitates deployment and recovery at sea. Because the data are transferred electronically to a microcomputer for archiving, the instrument can be redeployed after use without opening the pressure case. Twenty to forty drops can be made before the battery needs to be replaced. Several different sensor configurations are possible, depending on the type of small-scale mixing process of interest. By observing both finestructure and microstructure with one instrument, it is possible to study the relationship between the finescale driving forces and the smallscale mixing events.
\end{abstract}

\section{Introduction}

Great strides have been made in the past decade toward understanding the nature of oceanic fine- and microstructure and their role in the thermohaline and mechanical energy budgets of the ocean. It is widely believed that the smallest scale features comprise the dissipation end of the ocean variability spectrum while the finestructure, with vertical scales from 1 to $100 \mathrm{~m}$ and horizontal scales around $1 \mathrm{~km}$, acts as a conduit, transferring variance downscale towards destruction. It has, however, been quite difficult to quantify the strength of these processes and to establish connections between phenomena on different scales. The procedures for sampling microstructure variability are complex and specialized equipment is required. As a consequence, microstructure instruments have often focused only on the smallest scales of variability. Thus, the relationships of mixing phenomena to behavior on other scales are generally poorly understood. A particularly relevant example of this problem is the work of Gargett et al. (1981) on describing the spectrum of shear variability. Data from no less than three separate instruments were used in constructing a composite spectrum. Such composition precludes the investigation of the joint behavior of different scales, on an event-by6413.

* Woods Hole Oceanographic Institution Contribution Number

Corresponding author address: Dr. John Toole, Woods Hole Oceanographic Institution, Woods Hole, MA 02543. event basis, since the space-time correlation scales for much of this variability are shorter than the physical and temporal separations between deployments of the different instruments.

Establishing a connection between motions on the fine- and microscales is vital to the parameterization of the effects of the small scale processes. Such parameterizations are, of course, absolutely essential, since theoretical models of the ocean require some representation of the mixing processes, and widespread direct sampling of the microscale is impractical. A significant step toward the realization of such parameterizations was taken by Schmitt and Georgi (1982). They found that the occurrence of microstructure was well correlated with both low Richardson numbers (Ri) and density ratios $\left(R_{\rho}\right)$ near one. This suggests that if the dependencies of the dissipation of mechanical energy $(\epsilon)$ and thermal variance $(\chi)$ on $R i$ and $R_{p}$ can be established, then quantification of finescale characteristics will be sufficient for determination of the effects of the microscale processes.

Some models are available which attempt to relate the distribution of Richardson number (Desaubies and Smith 1982; Toole and Hayes 1984) and density ratio (Schmitt 1981) to mixing rates. These work well at explaining the observed occurrence of $R i$ and $R_{p}$ in the ocean, but the vertical diffusivities derived from them will remain speculative until adequate measurements of both finestructure and microstructure can be made with one instrument. As noted in the review of Caldwell (1983), there exists a clear need for new instruments to sample the finescale shear, temperature and salinity fields and the microscale fields simultaneously, thus 
permitting exploration of the physics of mixing. In response to this need a new free-fall instrument, the High Resolution Profiler, (HRP) was designed and constructed at the Woods Hole Oceanographic Institution. The HRP is similar in design to the profilers TOPS (Hayes et al. 1983) and MSP (M. Gregg and T. Sanford, personal communication). The most important characteristics of the vehicle are

1) the ability to profile temperature, salinity and horizontal velocity from large scales down to vertical scales of order one meter, and

2) the ability to resolve microstructure in temperature, conductivity and velocity on centimeter scales in the vertical and detect centimeter scale conductivity variability in the horizontal.

To achieve these goals a flexible suite of sensors was incorporated into one package. Finescale information is obtained with a conventional Conductivity-Temperature-Depth instrument (CTD) and a two-axis acoustic current meter. Small scale temperature, conductivity and velocity sensors provide microstructure information in the vertical. Information about the horizontal variability of conductivity is provided by microsensors deployed on extended fins, which force the vehicle to rotate as it falls, sweeping these sensors through a helical path. Finally, an optional shadowgraph system (Converse et al. 1986) is available to record images of small scale index of refraction variations. Onboard accelerometers and magnetometers, used to monitor the body motion, round out the sensor suite.

In the following sections, details of the instrument are outlined. We have been guided by the experience accumulated by colleagues in the past decade and have actively consulted with the developers of existing profilers while building this instrument. Therefore, wherever possible, tried and proven techniques and commercially available sensors were utilized. Section 2 details the mechanical design of the profiler and its shipboard deployment system. Section 3 treats the sensors employed on the instrument, while section 4 provides a description of the microcomputer system developed for the profiler and outlines the operational software. Section 5 discusses the shipboard data processing system, and section 6 contains a presentation of the velocity data processing techniques. Finally, we conclude with examples of the data obtainable with this instrument.

\section{Mechanical design}

The basic design for the vehicle is very similar to several previous instruments in that it is a long thin cylinder (Fig. 1). The motivation for this stems from the vast experience with instruments of this shape and the recent advances that have been made in understanding the dynamics of these vehicles. The most so- phisticated instrument response model to date is that developed by Dr. S. P. Hayes and colleagues at the Pacific Marine Environmental Laboratory (Hayes et al. 1983, HMF hereafter) for their profiler, TOPS. We
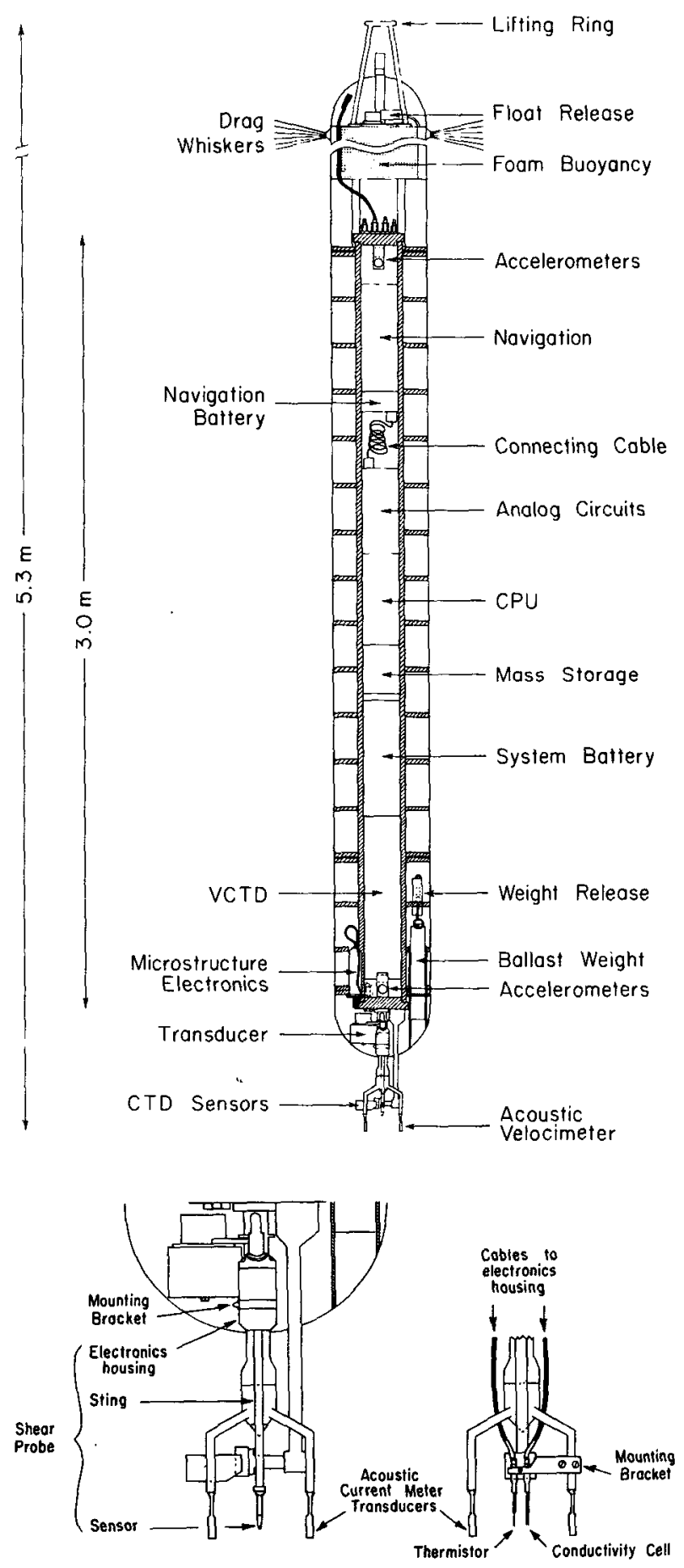

FIG. 1. (a) A schematic of the fine- and microstructure profiler. Note the break in scale at the upper end of the instrument. (b) Close up view of the lower end of the instrument with various sensors. 
have purposely designed the instrument to be similar in shape to TOPS to be able to easily transfer the HMF model to our instrument.

The HRP is $5.3 \mathrm{~m}$ in overall length (sensors to lifting bail); the main body is $38.1 \mathrm{~cm}$ (15 in) in diameter and $4.57 \mathrm{~m}$ long (Fig. 1). This extreme length was dictated by a requirement for profiler stability against tilting and to maximize the relative velocity signal. A standard $15.4 \mathrm{~cm}$ (6 in) ID, $18.4 \mathrm{~cm}$ (7.25 in) OD pressure case, $3 \mathrm{~m}$ in length, was used to house the bulk of the electronics. To permit exploration of the abyssal ocean and for safety considerations, the pressure case is able to withstand full ocean depth pressures $(6000 \mathrm{db})$. The instrument diameter is enlarged beyond the pressure case with a free-flooding external plastic shell supported by ribs spaced along the body of the pressure case. Hemispherical shells cover each end to provide smoother leading and trailing surfaces for the body. Syntactic foam, which provides buoyancy for the profiler, fills the shroud at the upper end as depicted in Fig. 1a. The foam and lifting bail are secured to the pressure case with four titanium rods in an arrangement which places the foam under compression, increasing the body's resistance to lateral flexing (important during recovery). Ballast release mechanisms, microstructure preamplifier packages and an acoustic transducer fit under the shroud at the leading edge. The placement of the transducer at the lower end facilitates tracking of the instrument when it is at the surface. Recovery aids, a light, radio and flag, are located at the top end.

The mass of the solid structure of the profiler is 274 $\mathrm{kg}$. The syntactic foam and pressure case provide sufficient buoyancy in sea water to support $272 \mathrm{~kg}$; hence on deployment the instrument is $19.6 \mathrm{~N}(2 \mathrm{~kg} \times 9.8$ $\mathrm{m} \mathrm{s}^{-2}$ ) negatively buoyant. Expendable ballast weights affect instrument recovery. Two independent weights (7.26 kg steel cylinders), each with redundant release mechanisms, are used to ensure recovery. Release of one weight is sufficient, however, both are normally dropped. Primary weight release is by a solenoid mechanism activated on command of the onboard instrument computer. Release is initiated when the profiler exceeds a preprogrammed pressure, or the dive exceeds a preset time interval. Two independent clocks time the dive. Hydrostatic shear pin releases and magnesium corrosible links provide backup for weight drop.

The main sensor suite is located at the leading (lower) end of the profiler (Fig. 1b). Separate support struts or stings are provided for the acoustic current meter (ACM), the finescale conductivity and temperature sensors, and the velocity microstructure probes. Temperature and conductivity microstructure probes are clamped to one of the ACM stings. A port for the pressure sensor is located in the lower end cap. Bulkhead connectors are mounted in both upper and lower end caps for electrical connections to microstructure preamplifiers, release solenoids, transducer and shipboard support computer.
The main instrument electronics are mounted in rack assemblies attached to each end cap and interconnected by multiconductor cable. Orthogonal accelerometer pairs are also mounted on the end caps. Two sets of accelerometers permit an independent estimate of the rate of change in instrument tilt because of their different positions relative to the center of gravity of the vehicle (section 6).

Several different configurations of the instrument are possible, as depicted in Fig. 2. These different deployment modes address a variety of problems in ocean microstructure. The first two involve changes in the configuration of elements at the top of the profiler, the last uses a special optical system attached at the bottom. The modes are:

1) Basic, in which drag elements at the top of the profiler serve to slow and stabilize the descent of the vehicle (Fig. 2a). Slight asymmetries in the drag elements force a slow rotation of the profiler during descent.

2) Rotating, in which four folding wings open when the instrument enters the water (Fig. 2b). Two of the arms are instrumented with microconductivity sensors for the detection of horizontal gradients as they trace out a helical path through the water.

3) Shadowgraph, in which the optical shadowgraph system described by Converse et al. (1986) is attached to the bottom of the profiler (Fig. 2c). A mechanical pressure release mechanism separates the two instruments in the event that the shadowgraph system is accidentally carried past its crush depth $(2500 \mathrm{~m})$.

The "basic" mode is the one which has been used most often, as it provides the simplest body motion and is easiest to handle on deployment and retrieval. In the "rotating" mode the arms lie alongside the body of the instrument on deck and when the instrument is rising. Once the instrument enters the water the arms deploy due to their slight buoyancy and the drag on the arms. The fin angle at the ends of the arms is 50 degrees. The azimuthal speed $\left(V_{a}\right)$ of the sensor at the end of the arm is given by

$$
V_{a}=\omega r
$$

with $\omega$ equal to the rotation rate of the vehicle about its long axis. With the arms deployed $(r=1 \mathrm{~m})$ the instrument spins about once every 16 seconds, yielding $V_{a}=0.39 \mathrm{~m} \mathrm{~s}^{-1}$. In this mode the fall rate is $0.48 \mathrm{~m}$ $\mathrm{s}^{-1}$; this compares to a fall rate of $0.60 \mathrm{~m} \mathrm{~s}^{-1}$ when the instrument is deployed with drag whiskers alone. The total speed of the conductivity probes at the arm tips is $0.62 \mathrm{~m} \mathrm{~s}^{-1}$; the angle of attack achieved is $39^{\circ}$ from vertical. The arm length was chosen to ensure that the sweep sensors are removed from the instrument boundary layer. The arms themselves are detachable to facilitate replacement of broken sensors and/or arms and permit return to the basic mode.

The shadowgraph system is described by Converse 

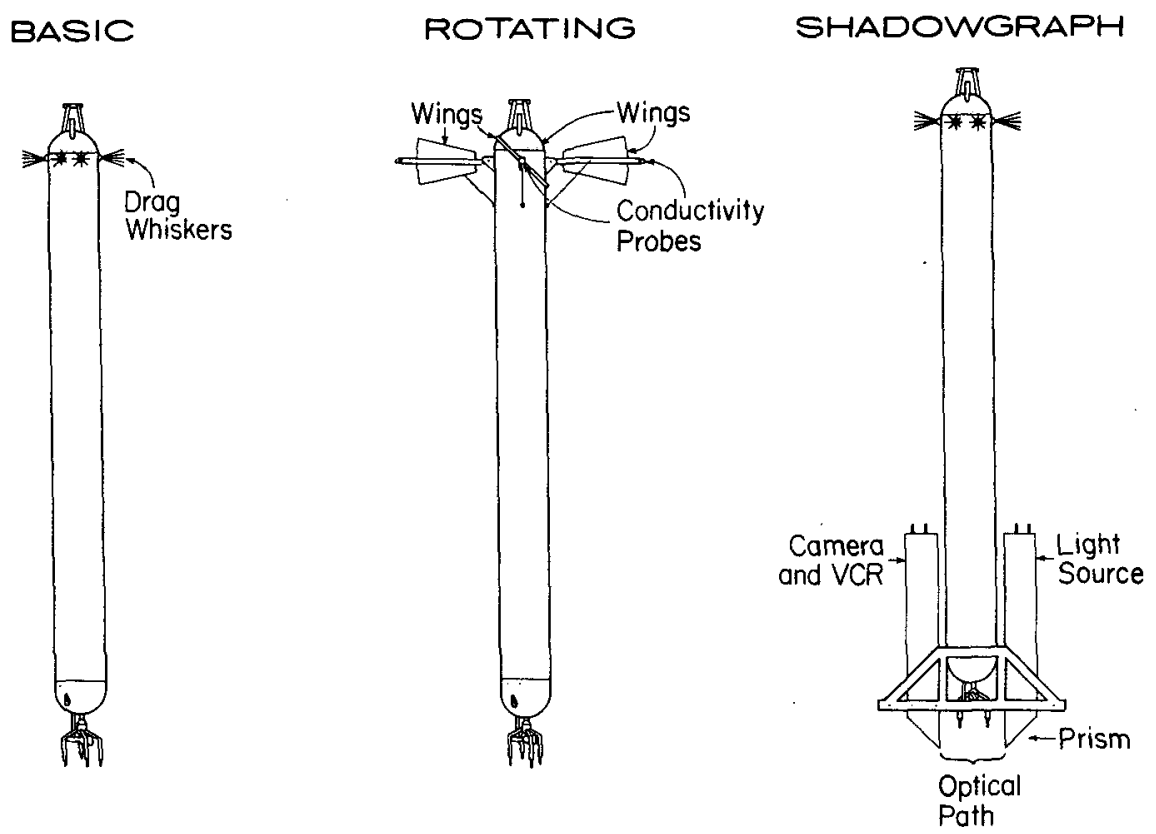

FIG. 2. (a) Profiler with drag whiskers. (b) Profiler with wings to induce rotation. Two of the wings are instrumented with microconductivity cells. (c) Profiler with optical shadowgraph system.

et al. (1986). It has been deployed independently and shown itself to be of great value in determining the structures of small scale mixing patches (Schmitt 1987; Kunze et al. 1987). To date the shadowgraph and the profiler have been used independently, not as the unified system shown in Fig. 2c. Combined shadowgraph/ HRP casts will be valuable for quantifying the response of the optical system.

The long body used for the HRP, while providing stability to tilting, also makes for an instrument that is potentially difficult to handle, especially since the sensors are quite fragile. We have dealt with this problem by designing a special handling rig that lowers and raises the profiler into/out of the water and tilts it to a horizontal position for on-deck servicing and storage between dives.

The handling rig consists of a deck-mounted track on which a wheeled cradle holding the profiler can ride (Fig. 3). The cradle itself mates with a hydraulically operated fork at the edge of the deck (the stern on Oceanus class vessels) which tilts the cradle vertically. The weight of the profiler is taken up by a line attached to a hydraulic winch mounted on the fork lift. The power pack for the hydraulics is located remotely to minimize noise on deck. The cradle pivots 10 degrees past vertical allowing the profiler to swing clear of the lower cradle arms. It is subsequently lowered into the water and released. For recovery, the HRP is ap-

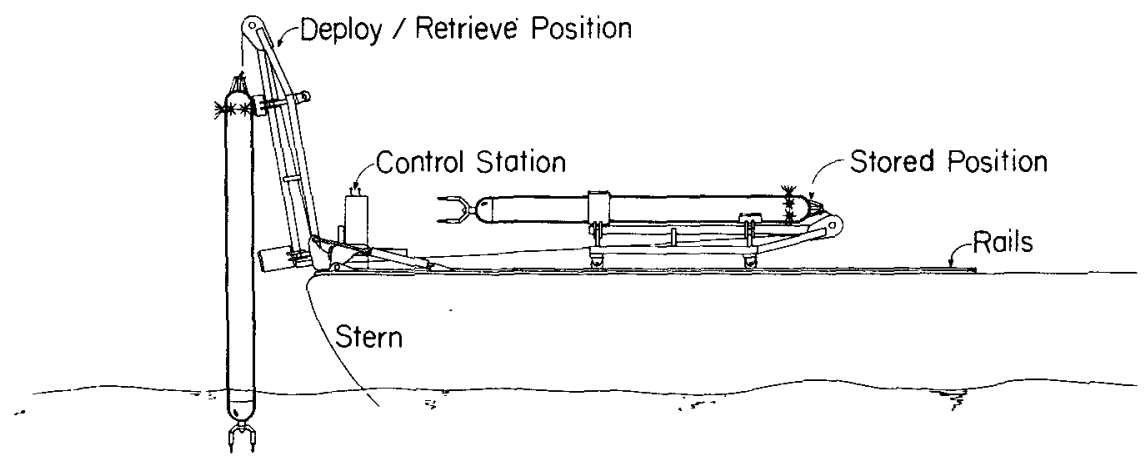

FIG. 3. The handling rig for the fine- and microstructure profiler. The instrument cradle rides on deck mounted rails. The profiler is stored and serviced with the cradle retracted from the edge of the deck; it is deployed and retrieved by tilting the cradle with a hydraulically operated pivot and winch mounted at the edge of the deck. 
proached by the ship so that a line can be attached; no small boat operations are necessary. Then the profiler is led astern, the lifting line secured, and the instrument is raised and tilted back to a horizontal position. We found that the great length of the instrument was, in fact, a significant advantage, in that the lower end of the instrument was submerged before the profiler left the cradle. This stabilized the profiler so that both launches and recoveries were well controlled. The length of the profiler also meant that accidental contact between the profiler and the ship caused only cosmetic damage to the profiler, as the sensors are well below the depth of the bilge keel on Oceanus class vessels. Routine operations are possible in fully developed seas resulting from $20 \mathrm{knot}$ winds, and a few recoveries have been done safely in higher winds (to $35 \mathrm{knots}$ ) before the seas built up. (A video tape of the operation is available from the authors upon request.)

\section{Sensors and electronics}

The profiler utilizes a large suite of sensors to sample finescale velocity, temperature and salinity structure and microscale temperature, conductivity and velocity variability. Selection of components was guided by performance specifications, availability, proof of reliability at sea, familiarity and compatibility with existing equipment, as well as expense. All finescale sensors are digitized at 16 bit resolution over oceanographic ranges and sampled at $10 \mathrm{~Hz}$. With a typical fall rate of 0.6 $\mathrm{m} \mathrm{s}^{-1}$ the vertical resolution is $6 \mathrm{~cm}$. The microstructure channels are also digitized at 16 bits and sampled at $200 \mathrm{~Hz}$, giving a vertical spacing of $3 \mathrm{~mm}$ between samples. Table 1 summarizes the finescale sensors and acoustic navigation equipment employed in the profiler. Each of the sensors is discussed in following separate subsections.
Power for all instrument electronics is supplied by four alkaline battery packs located in the lower half of the main pressure case. The normal power requirements amount to about 0.6 ampere-hour $(\mathrm{AH})$ for a $1000 \mathrm{~m}$ dive; this is drawn from three of the packs with a total capacity of $24 \mathrm{AH}$. A separate $8 \mathrm{AH}$ battery is reserved for the acoustic transducer and weight releases. We have found that it is possible to perform 30 to 40 drops on one set of batteries.

\section{a. Acoustic current meter}

A design goal for the profiler was the resolution of horizontal currents on vertical scales down to $1 \mathrm{~m}$ with sufficient accuracy to estimate the Richardson number. An essential element in accomplishing this is the 2axis acoustic current meter (ACM) at the lower end of the instrument. Section 6 describes how flow measurements relative to the body are used to deduce the larger scale velocity field. The profiler utilizes a Neil Brown Instrument System (NBIS) current meter, which has a $20 \mathrm{~cm}$ acoustic path length and operates at 2.75 $\mathrm{MHz}$. This sensor was chosen because it has proven reliable on the profilers TOPS (HMF), Yvette (Evans et al. 1979) and MSP (M. Gregg and T. Sanford, personal communication, 1986). On the advice of S. Hayes extra potting material was added around the transducers to prevent leaks. Figure 4 presents a frequency spectrum of data from the ACM from a cast in the main thermocline of the Sargasso Sea. From the flattening of spectral levels at frequencies above $2 \mathrm{~Hz}$, we estimate the noise level for this sensor as $\pm 0.065 \mathrm{~cm}$ $s^{-1}$. This is above the quantizing noise $\left[\left(a^{2} / 12\right)^{1 / 2}\right.$, where $a=$ least bit; Bendat and Piersol 1971], indicating that the $\mathrm{ACM}$ may require further tuning or filtering to fully warrant digitization to 16 bits.

Calibration of this sensor involves two 'separate problems: flow distortion and sensor bias. From mod-

TABLE 1. Finescale sensors.

\begin{tabular}{|c|c|c|c|c|c|}
\hline Sensor & Manufacturer & $\begin{array}{l}\text { Rate } \\
(\mathrm{Hz})\end{array}$ & Range & Resolution & Noise \\
\hline Acoustic current meter & $\begin{array}{l}\text { Neil Brown } \\
\text { Instrument Systems }\end{array}$ & 10 & $\pm 1.15 \mathrm{~m} \mathrm{~s}^{-1}$ & $3.5 \times 10^{-5} \mathrm{~m} \mathrm{~s}^{-1}$ & $\pm 6.5 \pm 10^{-4} \mathrm{~m} \mathrm{~s}^{-1}$ \\
\hline Platinum thermometer & $\begin{array}{l}\text { Neil Brown } \\
\text { Instrument Systems }\end{array}$ & 10 & $0-32^{\circ} \mathrm{C}$ & $0.0005^{\circ} \mathrm{C}$ & $\pm 1.4 \times 10^{-40} \mathrm{C}$ \\
\hline Conductivity & $\begin{array}{l}\text { Neil Brown } \\
\text { Instrument Systems }\end{array}$ & 10 & $0-65 \mathrm{mS} \mathrm{m}^{-1}$ & $0.001 \mathrm{mS} \mathrm{m}^{-1}$ & $\pm 2.9 \times 10^{-4} \mathrm{mS} \mathrm{m}^{-1}$ \\
\hline Pressure & $\begin{array}{l}\text { Neil Brown } \\
\text { Instrument Systems }\end{array}$ & 10 & $0-6500 \mathrm{db}$ & $0.1 \mathrm{db}$ & $\pm 0.06 \mathrm{db}$ \\
\hline Magnetometer & $\begin{array}{l}\text { Neil Brown } \\
\text { Instrument Systems }\end{array}$ & 10 & $0-360^{\circ}$ & $0.04^{\circ}$ & $\pm 0.4^{\circ}$ \\
\hline Accelerometers & Sunstrand (QA-1400) & 10 & $\pm 1.0 \mathrm{~m} \mathrm{~s}^{-2}$ & $3.0 \times 10^{-5} \mathrm{~m} \mathrm{~s}^{-2}$ & $\pm 7.1 \times 10^{-4} \mathrm{~m} \mathrm{~s}^{-2}$ \\
\hline \multicolumn{6}{|c|}{ Navigation } \\
\hline $\begin{array}{l}\text { Acoustic } \\
\text { Travel Times (Round } \\
\text { Trip) }\end{array}$ & $\begin{array}{l}\text { EG\&G } \\
\text { Sea-Link }\end{array}$ & 0.05 & $\begin{array}{l}0-10 \mathrm{~s} \\
\text { (not tested to date) }\end{array}$ & $.02 \mathrm{~ms}$ & $0.4 \mathrm{~ms}$ \\
\hline
\end{tabular}




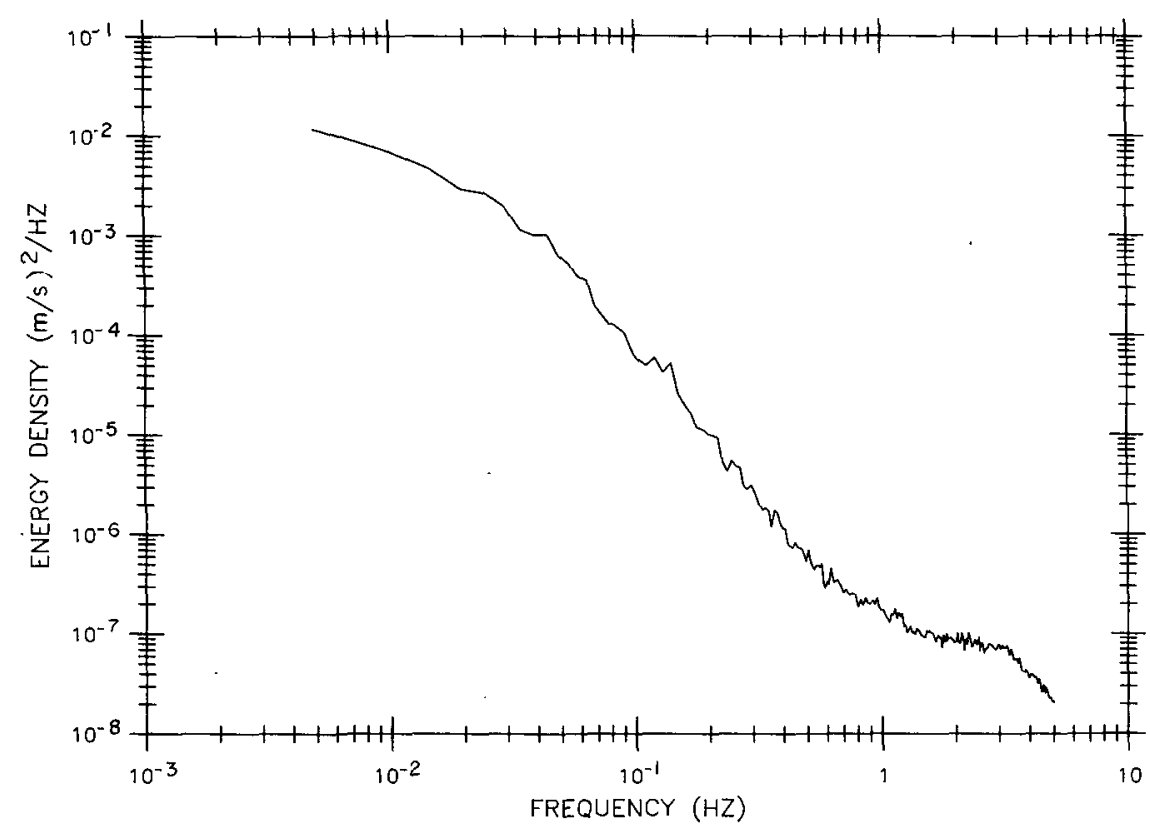

Fig. 4. Frequency spectrum of velocity measured by the acoustic current meter on the profiler. The instrument pendulum frequency is about $0.125 \mathrm{~Hz}$ where a slight dip followed by a small peak in energy density is observed.

eling work done for TOPS, it is estimated that the observed relative flow at the nose of the profiler is some $5 \%$ greater than the true relative flow because of streamline distortions (HMF). To date the flow acceleration has not been measured directly. The bias problem arises because the sensor zero point drifts with time, introducing errors of up to $0.5 \mathrm{~cm} \mathrm{~s}^{-1}$. Hayes et al. (1983) describe a scheme to determine the bias in the velocity data after acquisition which is feasible for the present system. The method involves estimation of the time-average velocity for each axis of the meter as the sensor rotates and falls through the ocean shear flow. Assuming the ocean shear is statistically stationary with depth, this average approaches the sensor bias.

\section{b. Conductivity-temperature-depth}

A one-meter resolution for temperature and salinity (thus density) was specified for the finescale CTD sensors on the profiler. The standard $0.1 \mathrm{db}$ resolution in pressure for these devices is sufficient to describe the near uniform fall rate of the instrument. The measurement of temperature and salinity is more problematic, as there is no direct means of obtaining water samples with the instrument for calibration; thus the temperature and salinity sensors must be very stable. With presently available technology, direct absolute temperature determination is feasible (calibration is stable for entire cruise). Conductivity sensors are more prone to drift. Even so, with careful handling, pre- and post cruise calibrations, and nearby CTD casts into water with stable $T-S$ characteristics, accurate absolute salinity can also be maintained (Luyten et al. 1982). The HRP is equipped with a Neil Brown Instrument Systems CTD. This unit has excellent spatial resolution and stability, is familiar to WHOI users and is compatible with in-house instrumentation. (In at-sea emergencies, spare electronics can be readily borrowed from CTD supplies, for example.)

The only modification we made to the standard CTD was to replace the platinum thermometer with a faster responding probe (also platinum, Rosemount model No. $171 \mathrm{CG}$ ). With the system's stable temperature calibration, determination of absolute salinity is relatively straightforward. During the one cruise performed to date the conductivity cell showed negligible drift. From the data in Table 1, it can be seen that the noise levels for temperature and conductivity are at the quantizing level; this reflects the high quality of the data obtainable from an internally recording vehicle free of ship motion and slip ring noise. There is a higher noise level in the pressure, which corroborates earlier experience with this strain gauge sensor (Georgi, Millard and Schmitt 1983).

\section{c. Accelerometers}

Two pairs of accelerometers, mounted orthogonally at the top and bottom of the instrument pressure case, are used to monitor body tilts. They are oriented to sense normal to the long axis of the profiler body. The use of pairs of sensors at both ends of the profiler provides a means of decoupling the measurement of tilt from the accelerations of the body (see section 6). Fol- 
lowing TOPS' lead we utilized Sundstrand servo accelerometers (model QA-1400). The calibration of the sensor gain is provided by the manufacturer, and $0.01 \%$ resistors are used in the amplifier circuitry. However, the zero point cannot be easily specified since the sensor mounting may not be perfectly horizontal. Here again, actual data is used to establish the zero by assuming that the time-average attitude of each accelerometer is horizontal. TOPS accelerometer measurements were affected by noise from inadequate anti-aliasing filtering and vibrations from the drive motor of its internal tape recorder, a problem eliminated by our solid state memory (see below). The least bit of our system corresponds to $3.0 \times 10^{-5} \mathrm{~m} \mathrm{~s}^{-2}$ in acceleration. Accelerometer frequency spectra are given in Fig. 5. The spectra go flat at about $0.5 \mathrm{~Hz}$ at a level of $1.0 \times 10^{-7}$ $\left(\mathrm{m} \mathrm{s}^{-2}\right)^{2} / \mathrm{Hz}$. Integrating to the Nyquist $(5 \mathrm{~Hz})$ indicates a noise level of $7.1 \times 10^{-4} \mathrm{~m} \mathrm{~s}^{-2}$, which is significantly better than the level of $7 \times 10^{-3} \mathrm{~m} \mathrm{~s}^{-2}$ we estimate for TOPS, though not as good as expected from the manufacturers specifications. We suspect that there is insufficient common mode rejection in our amplifier circuit which might be improved with slight modifications.

\section{d. Magnetometer}

A two-axis magnetometer is required to monitor the azimuthal orientation of the profiler for later separation of the horizontal currents into east and north com- ponents. Like TOPS we utilized the NBIS unit that is an integral part of the acoustic current meter. Calibration of the sensor is accomplished in a manner similar to that used to define bias in the acoustic current meter and accelerometer. As the profiler rotates, each component of the magnetometer cycles through \pm full scale each revolution. A plot of one component versus the other must therefore define a circle with origin at $(0$, $0)$. Offsets and the scaling for each component are selected to force this to be the case.

\section{e. Acoustic tracking}

The acoustic tracking system utilizes an array of bottom mounted transponders to track the three dimensional position of the profiler during its descent. The time derivative of the horizontal position of the profiler yields a long wavelength estimate of the horizontal velocity field as described by Luyten et al. (1982). Absolute accuracy depends on the errors in surveying the transponder array and in the resolution of the travel time measurement. Following the lead of White Horse and TOPS, we have equipped the profiler with an AMF tracking system. The sampling is controlled by the onboard microprocessor (see below). It is presently programmed to record round-trip travel times for three different acoustic frequencies near $10 \mathrm{KHz}$ every 20 seconds. The resolution is $0.2 \times 10^{-4} \mathrm{~s}$. Our estimate of the noise is based on past experience with these systems, since this part of the profiler has not yet been

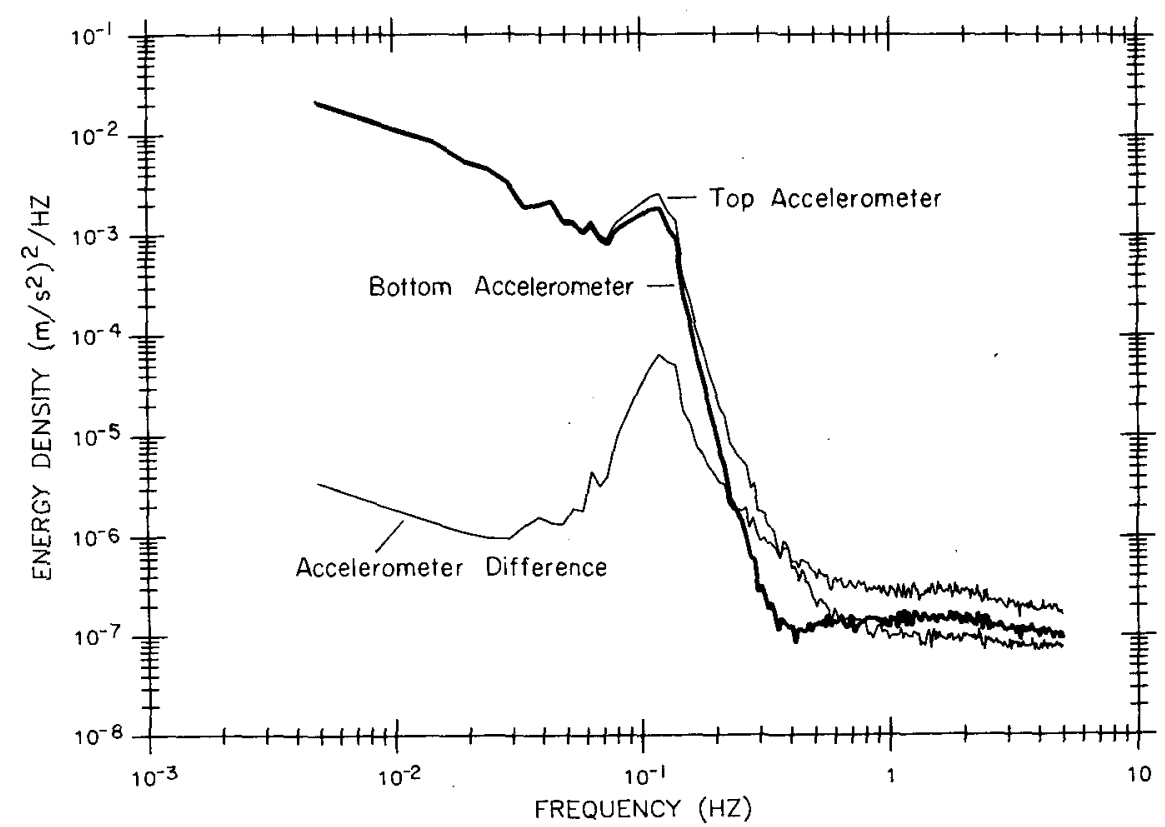

FIG. 5. Frequency spectra of accelerometer data from sensors located at the lower end cap (bold line) and upper end cap (thin line). The two spectra diverge about the instrument pendulum frequency of $0.125 \mathrm{~Hz}$. Also shown is the spectrum of the difference between top and bottom accelerometers which is sharply peaked at the pendulum frequency. Sensor noise levels are reached at around $0.4 \mathrm{~Hz}$. 
tested because of the failure of expendable transponders during the first cruise.

\section{f. Microstructure sensors}

While the appropriate finescale sensors have been on the market for some years, the techniques for measuring microstructure are more specialized, and it is only very recently that commercially produced sensors have become available. Where possible we employed these new sensors, rather than fabricate our own, in order to cut development time.

Three different microstructure sensors can be used with the profiler: 1) microbead thermistor, 2) dual electrode microconductivity cell, and 3) airfoil shear probes. Each microstructure probe has an associated selfcontained electronics package which houses circuitry to amplify and preemphasize the signal from the sensors. These small pressure vessels are connected to the actual probes via rigid stings in the case of the shear probes and flexible oil-filled cables for the thermistor and conductivity cells (see Fig. 1b). The packages are connected by cable to the electronics in the main pressure case which supplies power and receives and digitizes the signal voltages.

The temperature microstructure sensor assembly, purchased from Sea-Bird Electronics, Inc., utilizes an FP07 thermistor. The use of microbead thermistors to sense oceanic temperature microstructure has been well documented (Lueck et al. 1977; Gregg et al. 1978; Gregg and Meagher 1980; Lueck and Osborn 1980; Lueck and Vachon 1984). While thermistors have a relatively strong response to temperature changes, their use as microstructure sensors is constrained by a finite response time which is a function of the glass coating and fluid boundary layer around the bead. A correction to temperature microstructure data is typically applied when estimating the temperature dissipation rate $(\chi)$ or the Cox number (Osborn and Cox 1980). The correction for the FP07 is discussed by Gregg et al. (1986).

The vertical wavenumber spectra of oceanic temperature, conductivity and velocity exhibit a steep negative slope with increasing wavenumber. Consequently, information at high wavenumbers (the focus of microstructure studies) is sensitive to errors deriving from the finite resolution of digitizers and electronic noise. The dynamic range of the microtemperature signals was compressed by preemphasizing the high frequencies prior to digitization in order to reduce truncation error and improve the signal to noise ratio. The filters employed provide unity gain at low frequency, permitting simple checks on sensor functionality, and resemble first-derivative operators for frequencies above $0.1 \mathrm{~Hz}$. The filter transfer function in frequency space $R(f)$, where $f$ is the frequency (in radians), is

$$
R(f)=(0.634+\text { if }) /\left(1.27 \times 10^{3}+\text { if }\right) .
$$

An anti-aliasing filter (4-pole Butterworth with $-3 \mathrm{db}$ point at $100 \mathrm{~Hz}$ ) dampens energy above the Nyquist frequency.

A recursive digital filter was devised to remove the preemphasis applied to the micro- temperature and conductivity channels. The transfer function of the required filter is simply the inverse of that given in Eq. (1). A recursive filter:

$$
y_{n}=a_{0} x_{n}+a_{1} x_{n-1}+b_{1} y_{n-1},
$$

where $x$ is the input time series and $y$ the output series with $n$ the scan number index, was employed. The coefficients were estimated by performing a bilinear transformation (Childers and Durling 1975) on the function $[1 / R(f)]$ and equating terms with the Z-transform of Eq. (2).

Calibration of the resulting high resolution temperature data was achieved with in situ data from the CTD. Local regressions between temperature sensed by the CTD and filtered microstructure data were performed yielding calibration formulae for the microtemperature series. For the data processed to date, running linear fits across five decibars of data were employed.

A recently developed microconductivity sensor complements the fast thermistor probe. The cell, (also purchased from Sea-Bird Electronics, Inc.) is described by Meagher et al. (1982); it consists of two freely flushing parallel platinum needles separated by $3 \mathrm{~mm}$. Meagher et al. (1982) have established that the $-3 \mathrm{db}$ point of this probe is at about $100 \mathrm{cpm}$. Electronic preemphasis of the microconductivity signal is also performed using the same filter that is applied to the temperature channel. Recursive filtering and calibration of the microconductivity data follow that for temperature. Regression against CTD derived conductivity yields a high resolution conductivity series potentially suitable for salinity calculations (in combination with the microtemperature data). In many applications, however, data from the microconductivity channel is interpreted as a measure of small scale temperature variability. Although conductivity is in general a function of salinity, temperature and pressure, on scales larger than $1 \mathrm{~cm}$ the contribution of temperature tends to dominate the measured conductivity. This is demonstrated in Fig. 6, where theoretical predictions of temperature, salinity and conductivity gradient spectra, based on the Batchelor (1959) theory, are given for a kinetic energy dissipation rate of $1 \times 10^{-9} \mathrm{~W} \mathrm{~kg}^{-1}$. Salinity significantly affects the shape of the conductivity spectrum only on scales smaller than $1 \mathrm{~cm}$ which is less than the resolution of the probe. For application as a temperature probe, the microconductivity are "calibrated" by local regression between the filtered microconductivity series and the CTD derived temperature data. Because the conductivity sensor has better spatial resolution than the FP07 thermistor at the profiler fall speed, thermal dissipation rates and Cox numbers derived from the microconductivity channel are felt to be more reliable than those from the micro- 


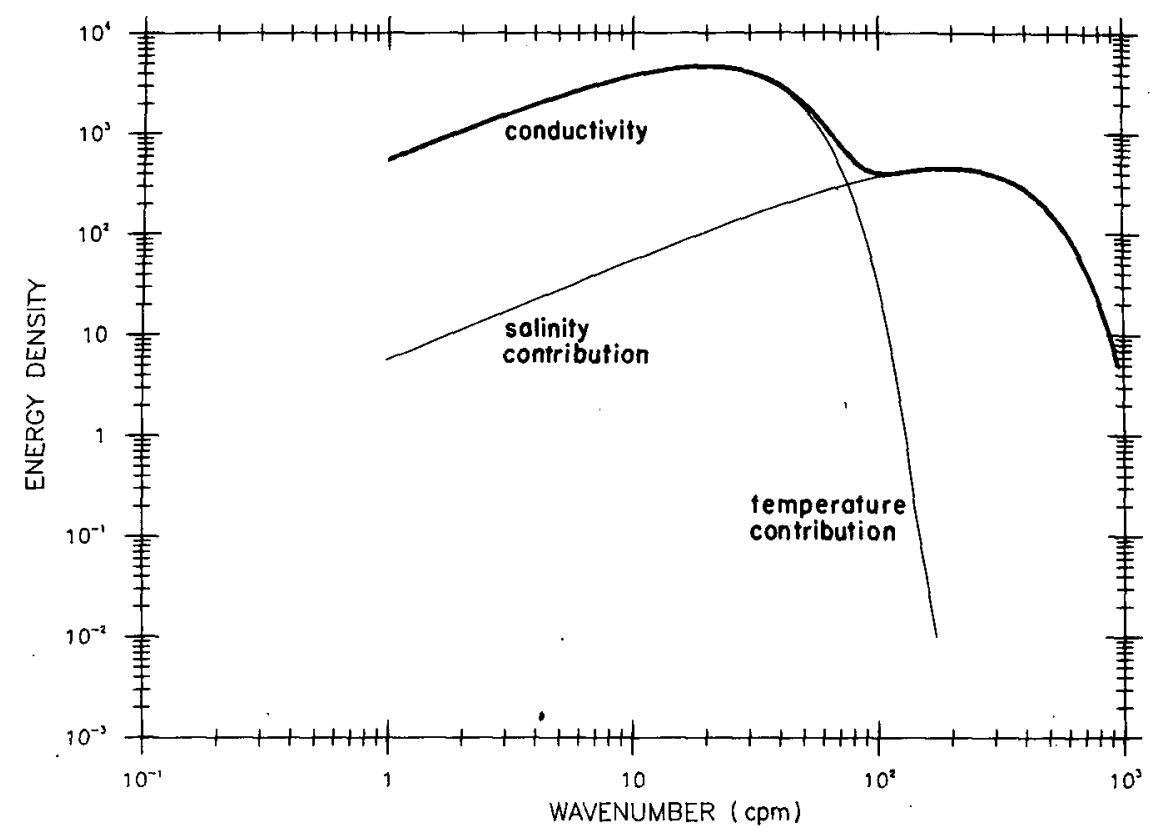

FIG. 6. Contributions of temperature and salinity variance to the spectrum of conductivity. The theory of Batchelor (1959) is used to predict the levels of scalar variance based on their different molecular diffusivities and a kinetic energy dissipation rate of $1 \times 10^{-9} \mathrm{~W} \mathrm{~kg}^{-1}$.

temperature sensor. No correction for probe response is required at moderate to low dissipation rates. Data from the microtemperature probe are preferred for calculation of the Thorpe Scale (Dillon 1982), where the results are less sensitive to structures on scales affected by the probe's limited spatial resolution.

Analysis of the noise characteristics of the Sea-Bird microstructure sensors indicates a frequency spectral energy density behavior as $f^{1}$ where $f$ is frequency. A noise variance estimate obtained by integrating this noise spectra to the Nyquist frequency $(100 \mathrm{~Hz})$ yields values of order $8.0 \times 10^{-5}{ }^{\circ} \mathrm{C}^{2} \mathrm{~m}^{-2}$. A spectral method for estimating dissipation rates where the wavenumber integration is cut off at an optimal wavelength to exclude the noise contribution to the variance estimate is discussed by Gregg et al. (1986).

Airfoil type shear probes (Osborn 1974; Osborn and Crawford 1980) have also been implemented to measure the dissipation of turbulent kinetic energy. Dr. N. Oakey, Bedford Institute of Oceanography, assisted with the development and loaned the probes for our first cruise. The sensor electronics are modeled after those of Oakey (1977); calibration and data reduction techniques follow his methods. The data from the initial deployments of these probes included contributions from vibrations at frequencies of $50-60 \mathrm{~Hz}$. Laboratory tests have since identified the chief offenders (primarily the conductivity-temperature sting for the CTD). Fortunately, these vibration noise signals lie at frequencies above the cutoff for the geophysical signal for moderate to low dissipation rates (Fig. 7). For the analysis of data recovered to date, a $15 \mathrm{~Hz}$ low-pass filter was applied to the raw data. The dissipation noise level for the resulting shear probe data, estimated from minimum observed levels, is about $4 \times 10^{-10} \mathrm{~W} \mathrm{~kg}^{-1}$. Design of a modified sensor support structure which should ameliorate the vibration contamination is underway.

The profiler normally carries a suite of vertically directed microstructure sensors consisting of one thermistor, one microconductivity cell and two shear probes. In the Rotating mode of deployment (Fig. 2), two additional microconductivity probes are mounted on horizontally deployed wings which force the vehicle to rotate. Mounted on orthogonal arms, these record data on a helical path and thus resolve horizontal gradients which are undetected by vertically directed sensors. This capability is of interest in ascertaining the isotropy of microstructure and should be particularly helpful in detecting salt fingers.

\section{Control processor}

The processor system used by the profiler was designed in cooperation with several other projects at the WHOI Applied Engineering Laboratory. The processor is based on the IEEE-796 (Multibus) backplane standard (Mellinger et al. 1986). Along with the new backplane and packaging specification, a family of 80C86based computer boards was designed, including the CPU, DMA and interrupt control, 64k memory, serial and parallel $\mathrm{I} / \mathrm{O}$, clock, timebase, and counter/timers, and a 16-bit optoisolated A/D converter board. 


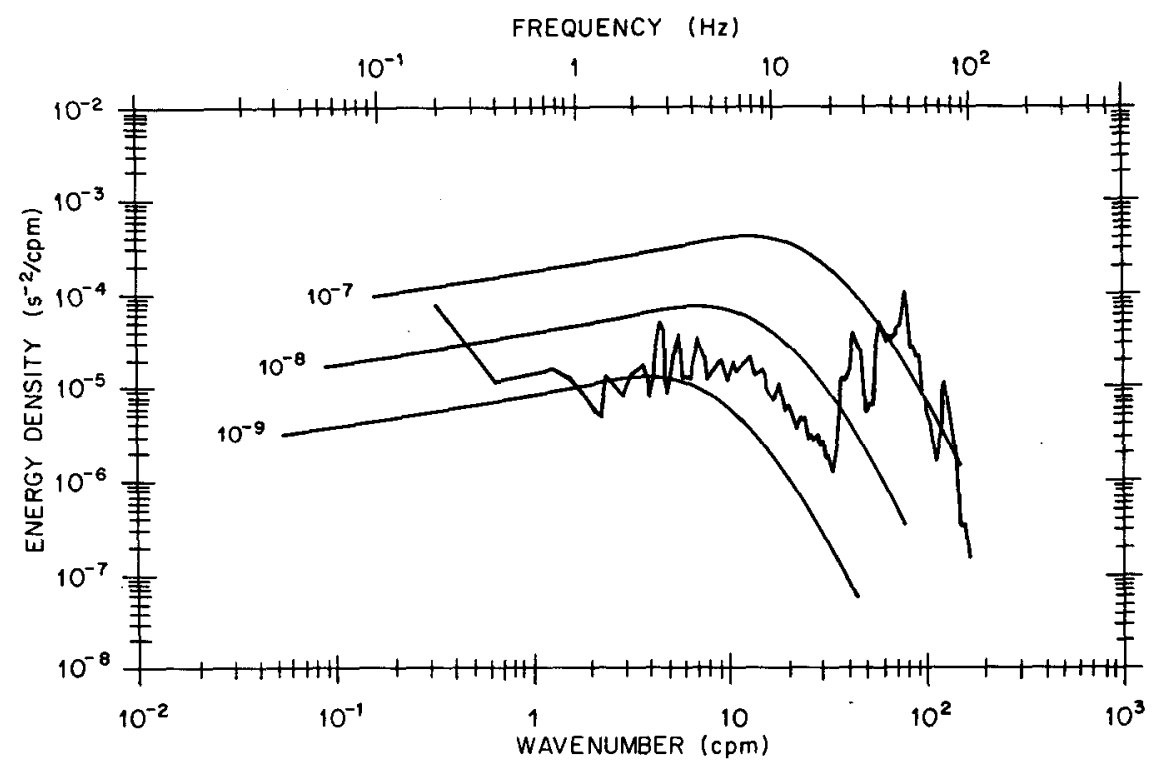

FIG. 7. Power spectrum of the raw signal from a shear probe on the profiler. The energy in the peaks at frequencies of $30 \mathrm{~Hz}$ and above are due to vibrations of the sensor stings. The data are low pass filtered with a cutoff at $15 \mathrm{~Hz}$ prior to further processing. Shown for reference are the universal shear spectra of Nasmyth after Oakey (1982) for the dissipation rates $\left(\mathrm{W} \mathrm{kg}^{-1}\right)$ shown in the plot.

The control processor configured for the profiler uses the $80 \mathrm{C} 86$ (at $5 \mathrm{MHz}$ ) with $64 \mathrm{k}$ each of RAM and EPROM (32k actually used to date). The CTD is interfaced through a custom clocked serial I/O board, with the balance of the sensors multiplexed into the A/D converter. A parallel I/O board provides bitwise functions such as power supply and weight release control.

Communication with the outside world is by current loop following the SAIL protocol. The link is used to configure the instrument before a dive and to unload data after a dive. Data transfer is 8-bit binary, serial asynchronous. At the present time software and hardware considerations limit speeds to 9600 baud $(\sim 1$ $\mathrm{kbyte} / \mathrm{sec}$ ), leading to an unpleasantly long total dump time of order two hours. An Ethernet based link is being developed to overcome this "bottleneck." This should result in data dump times of less than $10 \mathrm{~min}$ utes.

Software for the profiler control processor was written in Computer Innovations " $\mathrm{C}$," with time critical routines coded in assembler. A ROM-resident MSDOS emulator makes portions of the instrument I/O environment look like an IBM PC to an executing program. This allows operating software to be edited, compiled, and initially debugged on a desktop computer, before being downloaded (via SAIL link) to profiler RAM for final testing. EPROMS are burned only after software development is complete. A separate ROM-resident monitor provides the download function, along with an extensive set of processor and sensor diagnostic, test, and calibration routines.
Profiler operations for each dive are specified by the user via menu-driven instruction sequences. The user selects which finescale and microscale sensor channels to sample, the pressure at which ballast weight is to be released, and the pressure interval for microstructure sampling. Finescale sampling commences a predetermined time interval after profiler deployment and is terminated at the maximum depth of the dive. Sensor selection and sampling depths are dictated by scientific interest under the constraint of available data storage.

Mass storage. The profiler mass storage subsystem uses 64 kbit RAM chips arranged in groups of 16 (128 kbytes) on Single-Inline-Package (SIP) modules. The SIP modules were developed especially for this application in cooperation with Advanced Electronic Packaging, Inc. (Los Angeles, CA), and have since become a successful commercial product for that company. Eight SIP modules fit on each card. Four cards, each with a capacity of one megabyte, form the mass storage for the profiler (Mellinger 1986). The mass store, complete with backup battery and memory-mapped interface to the control processor, takes 6 inches of space inside the pressure case. The recent introduction of 256k static CMOS RAMs will allow 16 megabytes to fit into the same space if desired.

All sensors are sampled with 16 bits of resolution. Each finescale sensor is sampled at a rate of $10 \mathrm{~Hz}$. There are 11 finescale data channels yielding the aggregate data rate of 220 bytes per second. Additional channels for battery voltage and the zero point of the A/D converter (analog ground) may also be recorded if desired. At a nominal fall rate of $0.5 \mathrm{~m} \mathrm{~s}^{-1}$ over a 
$4000 \mathrm{~m}$ path, the total finescale data volume is 1.76 megabytes. The microstructure sensors, when active, are sampled at a rate of $200 \mathrm{~Hz}$. Up to six microstructure channels may be activated giving an aggregate data rate as high as 2400 bytes per second. Total microstructure data volume for all 6 channels over a $450 \mathrm{~m}$ aperture is 2.16 megabytes. The acoustic tracking data acquisition rate is low, a $5000 \mathrm{~m}$ drop yielding 2 kbytes of information. Use of the instrument in shallower dives is a more common deployment mode. When four channels of microstructure data are recorded, 3.2 megabytes of microstructure data are obtained in a $1000 \mathrm{~m}$ cast, along with less than 0.5 megabyte of finescale data. The microstructure, finescale and tracking data are stored in separate files in memory.

\section{Shipboard processing}

The shipboard computer system consists of a DEC Micro-VAX 1 computer with terminals, tape drives and plotter. Data processing and display programs are written largely in Fortran. This computer was chosen in part for compatibility with the WHOI main frame computers and the seagoing computers of the WHOI CTD group (Micro-VAX IIs), so that backup capability would be readily available. The Micro-VAX 1 has logged more than 2 months of operating time at sea, and 1 month of transit time with no hardware failures.

Data transfer and processing software. The first act after securing the profiler from a dive is to initiate the data transfer programs to extract the finescale, microstructure and navigation data files. The HRP transfer subroutine transmits data in 1024 byte blocks when prompted with the address of each block by the MicroVAX. A checksum is computed so that a block can be retransmitted in the event of error. The program can be restarted at the point of interruption if there is a break in the signal line. Three raw data files are generated on the Micro-VAX corresponding to the fine-, micro- and navigation data. The raw data is in the form of digitizer counts that are scaled to engineering units in a program, which allows for the introduction of slope and bias terms for all variables. Also, quadratic, cubic and pressure terms are used for the conductivity and temperature channels from the NBIS CTD. The data are stored in unformatted disk files with header information and time (scan number) as the implicit sequential variable in the form specified by Millard and Galbraith (1982).

Further processing for the various file types differs. The finescale CTD data are used to compute salinity, with the time scale mismatch of the thermometer and the CTD cell being corrected with a convolution filter that minimizes the phase shift between $T$ and $C$ (Horne and Toole 1980). Temperature and salinity are then pressure sorted into $0.5 \mathrm{db}$ bins; $8-10$ scans typically fall into each $0.5 \mathrm{db}$ interval. The accelerometer channels are used to provide a "de-nodding" correction to the velocity channels, which are used with the compass readings in a vehicle response model to obtain ocean velocity profiles (section 6 ). The resulting east and north velocity estimates are pressure sorted at $0.5 \mathrm{db}$ along with the temperature and salinity data, giving one file per dive suitable for the calculation of density, shear, Richardson numbers, etc. over scales larger than $1.0 \mathrm{db}$.

The microstructure data are handled differently. Quick estimates of temperature and velocity dissipation rates are obtained from the microstructure data in the time domain by exploiting the first difference nature of the preemphasis filters employed on these data channels. The shear probe data is low-passed to exclude vibrational noise and the variance of each channel is estimated in $0.5 \mathrm{db}$ windows aligned with the pressure sorted finescale data. Calibration factors are applied to estimate $\epsilon$. Similarly, the variance of the locally calibrated microtemperature and conductivity data yield $\chi$ estimates. Both the raw data time series and the dissipation profiles are used to evaluate sensor performance at sea. More detailed processing, which incorporates sensor response function corrections, is accomplished ashore.

\section{Velocity processing procedures}

As noted in the Introdution, measurements of flow with respect to the vehicle can be used to deduce the structure of the ocean velocity field. The transfer function between the relative flow and the ocean shear is a function of the dynamics of the body motion. This problem has been treated at great length by HMF. Drawing on their work we will describe an additional velocity correction technique made possible by the use of two pairs of accelerometers on our instrument (TOPS employed one pair of accelerometers). This approach allows us to estimate the tilt of the profiler so that a correction for such motion can be made.

We first consider the relation between the measured velocity from the acoustic current meters $U_{s}$, the horizontal velocity of the ocean $V_{0}$, the velocity of the center of mass of the instrument $U_{E}$, and the tilt of the instrument away from vertical $\theta$. This is Eq. (24) of HMF:

$$
U_{s}(t)=\gamma\left[V_{0}(t)-U_{E}(t)-L_{v c} d \theta / d t\right]
$$

where $\gamma=1.05$ is a correction factor for the flow distortion near the front of the vehicle, and $\left(L_{v c}\right)$ is the distance between the velocity sensors and the effective center of gravity of the instrument. Note that the time varying tilt of the instrument enters as a contaminant of the velocity difference between the profiler's center of gravity and the ocean. This contamination can be evaluated by utilizing the accelerometers mounted on the endcaps of the main electronics housing. The signal measured by an accelerometer located a distance $L_{a c}$ 
from the instrument's center of gravity is given by equation 25 of $\mathrm{HMF}$ :

$$
a(t)=g \theta+d U_{E} / d t+W_{0} d \theta / d t+L_{a c} d^{2} \theta / d t^{2} .
$$

This expresses the dependence on 1) tilt in the gravity field (small angles assumed), 2) the horizontal acceleration of the center of mass of the profiler, 3 ) the "cornering" acceleration felt as the instrument changes direction while falling at the rate $W_{0}$ (this arises because the body centered coordinate system rotates), and 4) the "swinging" of the accelerometer about the center of gravity. Note that the accelerations measured at two different points along the profiler vertical axis will be different because of the last term in Eq. (2). For sensors located above or below the center of gravity of the instrument the swinging term is either the same sign (top) as the gravitational term or of opposite sign (bottom). Figure 8 illustrates this effect. The difference between the two is evidenced in the accelerometer frequency spectra for sensors at either end of the profiler (Fig. 5). At low frequencies, the accelerometer signals are dominated by the gravitational term (first term on the RHS or Eq. (2)) which is the same for each sensor, to within calibration error. The spectra diverge at about $0.1 \mathrm{~Hz}$

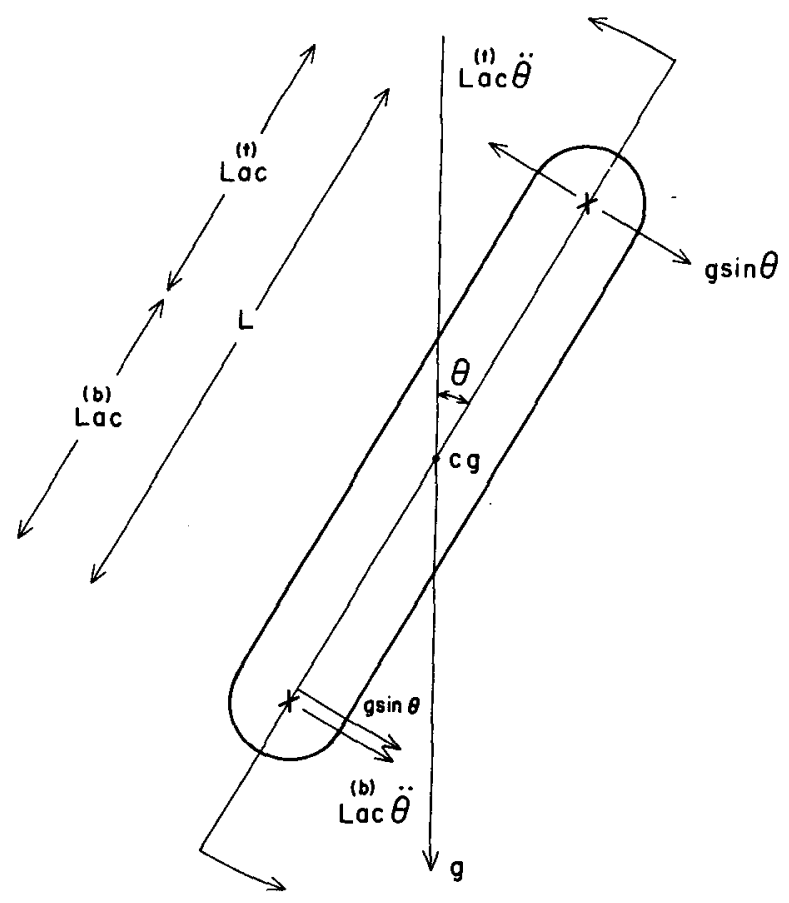

FIG. 8. Diagram of the accelerations experienced by points located at each end of the profiler. Consider the motion of pendulums (a good model of an accelerometer) located at each accelerometer site $(X)$. In the instrument attitude shown, the top pendulum is deflected to the right by the gravitational force. The acceleration of the top of the instrument to the left also causes the pendulum to deflect to the right relative to the instrument. Thus, the effects of gravitation and instrument tilting are additive in the measured acceleration at this site. Similar analysis of the lower site reveals that the terms partially cancel below the instrument center of gravity. with the energy density of the upper accelerometer (where the gravitational and swinging terms are additive) exceeding that of the lower (where gravity and swinging partially cancel) by about $2 \%$.

The difference of accelerations measured at the top $\left(a_{T}\right)$ and bottom $\left(a_{B}\right)$ of the profiler from Eq. (2) reduce to

$$
a_{T}-a_{B}=h d^{2} \theta / d t^{2}
$$

where $h$ is the distance between the two measurement points. Accelerometer difference spectra are peaked at a period of $8 \mathrm{~s}$, the pendulum period of the profiler, Fig. 5. Time integration of the accelerometer difference series yields a direct measure of the time rate of tilt of the profiler: a term which appears as a contamination of the measurement of $\left(V_{0}-U_{e}\right)$ in Eq. (1). Evaluation of the contamination term requires estimation of the two length scales, $h$ and $L_{v c}$. Direct measurement yields a value for $h(2.92 \mathrm{~m})$. The position of the effective center of gravity, $L_{v c}$, is computed in two ways. First, we use detailed estimates of the mass distribution in the profiler (as discussed by HMF); this gives a position of $2.57 \mathrm{~m}$ above the velocity sensors. Second, we examine the correlation of the integrated accelerometer difference time series with the measured velocities. That is, for each component of tilt, Eq. (3) gives

$$
d \theta / d t=\dot{\theta}=\frac{1}{h} \int\left(a_{T}-a_{B}\right) d t .
$$

Regression of $\dot{\theta}$ against $U_{s}$ for a data interval at the beginning of a dive (which typically exhibits strong swinging motion), yields $L_{v c}=2.48 \mathrm{~m}$, which is within the uncertainty of the computed position. The analysis utilizes the latter value.

Figure 9 demonstrates how the accelerometer data are used to remove the effects of profiler tilting from the measured velocity. The depth interval selected for display is characterized by a series of abrupt velocity changes with time. Torques about the instrument's center of gravity produced by relative horizontal flow cause the profiler to tilt. The tilts are not large however, (of order $1 / 2 \mathrm{deg}$ ) owing to the strong righting moment of the profiler and its large moment of inertia. As a consequence, the amplitude of the accelerometer derived velocity correction is small compared to the directly measured velocity. This characteristic is evident in spectra of measured velocity and acceleration (Figs. 4 and 5). Unlike the data obtained with TOPS, pronounced peaks in the velocity spectrum about the pendulum frequency are not observed (see HMF, Fig. 8). We attribute this difference to the greater length of the new profiler (body length, excluding sensor stings and lifting ring, of $4.57 \mathrm{~m}$ as compared to $3.06 \mathrm{~m}$ for TOPS) and hence greater effective moment of inertia (1227 $\mathrm{kg} \mathrm{m}^{-2}$ versus $884 \mathrm{~kg} \mathrm{~m}^{-2}$ for TOPS). Analysis in terms of the model developed by HMF indicates that the resonant tilting period of HRP is comparable to 


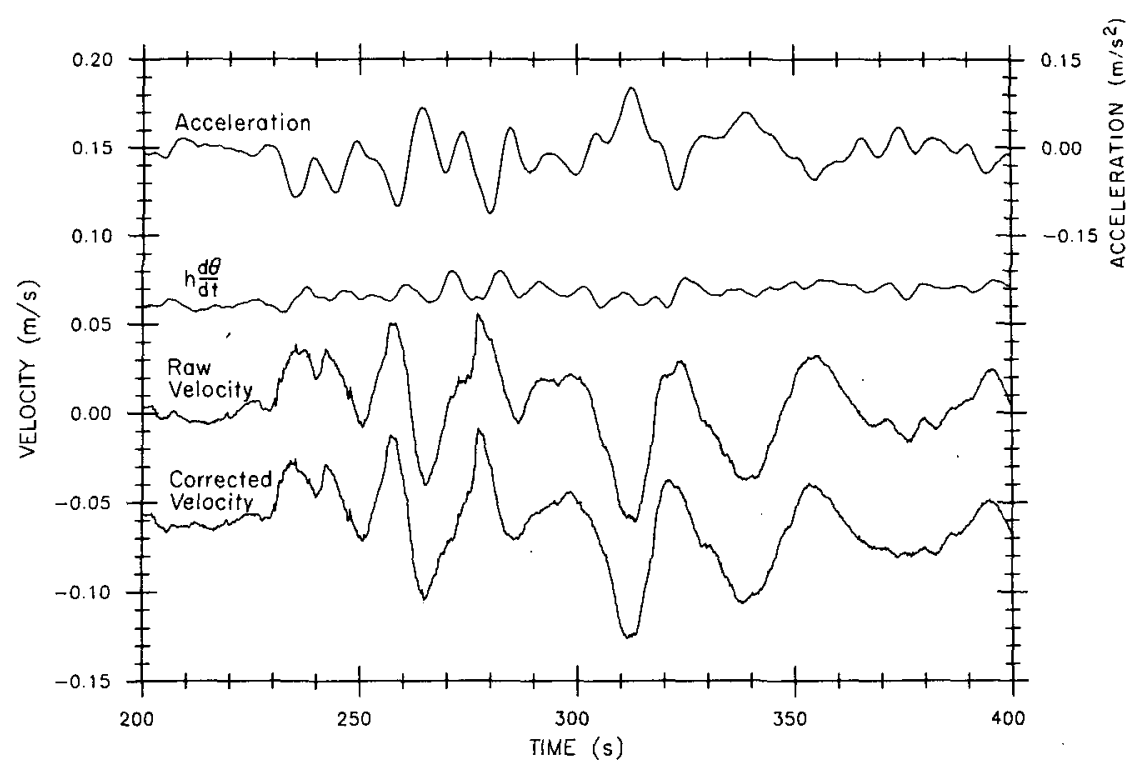

FIG. 9. Time series of accelerometer and acoustic current meter data from FASINEX. Shown are raw profilers of accelerations measured at the lower end cap and the velocity relative to the profiler in the same coordinate axis. The series of scaled integrated accelerometer difference constitutes the correction for instrument tilting. The "corrected" velocity profile is the difference between the raw velocity profile and the tilt-correction velocity.

the decay time of this mode of motion. Thus, tilts are more rapidly damped for the HRP than for TOPS, where the damping time is longer than the pendulum period. The raw velocity series (Fig. 9) exhibits several features which are correlated with the accelerometer derived velocity, such as those between time 350 and $400 \mathrm{~s}$. These are the result of the tilting of the instrument. The velocity series in which the tilting signal has been removed (by differencing the raw velocities and the integrated accelerometer difference time series) is visually smoother than the raw velocity series. The features between 350 and $400 \mathrm{~s}$ are substantially suppressed; structure associated with velocity peaks like those at 280 and $320 \mathrm{~s}$ are more continuous. By and large, however, the accelerometer-derived velocity corrections are subtle, owing to the small tilts.

Estimation of the oceanic velocity profile from the tilt-corrected relative velocity data is accomplished with the "point-mass" algorithm given by HMF and Evans et al. (1979). At periods long compared to the instrument pendulum period this model is equivalent to the more sophisticated model described by HMF. Use of the tilt-corrected relative velocity suppresses errors in the point-mass model at the pendulum period, so that use of the full HMF model is not warranted. The point mass model uses a simplified equation of motion for the profiler which in general relates lateral accelerations of the instrument to forces derived from relative horizontal flow between the instrument and the ocean. The model assumes that these forces are dominated by the force at the lower end of the profiler, $F_{n}$, which is evaluated using potential flow theory [Eq. (9) of HMF]:

$$
F_{n}=\pi a^{2} \rho W_{0}\left[V_{0}(t)-U_{E}(t)\right]
$$

where $a$ is the profiler body radius and $W_{0}$ the instrument fall rate. Including the effects of induced mass we have [Eq. (32) of HMF]:

$$
2 M d U_{E} / d t=\pi a^{2} \rho W_{0}\left[V_{0}(t)-U_{E}(t)\right] .
$$

In this relation $M$ is the mass of the profiler (including water trapped inside the instrument outer shell) or equivalently, the induced mass (the $2 \mathrm{~kg}$ difference between these is neglected). Expressing the induced mass in terms of the volume of the cylindrical profiler body and integrating in time yields

$$
U_{E}(t)=\int \frac{W_{0}(t)}{2 l} U_{c}(t) d t
$$

where $l$ is the instrument length. Here we have used the "corrected" velocity, $U_{c}=V_{0}-U_{E}=U_{s} / \gamma$ $+L_{v c} \dot{\theta}$ in place of $U_{E}$ since the point mass model is not strictly valid near the nodding period. Use of the corrected velocity in Eq. (9) is justified since the response time for horizontal motion of the instrument is long with respect to the nodding period of the profiler (the dominant signal of the velocity correction term). Given the above estimate of the motion of the instrument center of mass, Eq. (1) is solved for the ocean velocity at the depth of the acoustic current meter:

$$
V_{0}(t)=U_{c}(t)+U_{E}(t)
$$

relative to a depth independent constant.

As has been demonstrated by HMF, use of the point- 
mass model generates excellent velocity profiles on scales both larger and smaller than the body length. We believe that our tilt corrected velocities extend the validity of the point-mass model through the range of scales normally affected by the pendulum motion of the body. In order to illusrate this, velocity shear spectra constructed from profiles obtained from the point-mass model both with and without the tilt-correction appear in Fig. 10. The spectra are from two different depth intervals in the Sargasso Sea. The variation in energy level is due to the variation in background stratification; the shallower depth range is weakly stratified $18^{\circ} \mathrm{C}$ water. The spectra are consistent with the composite spectrum discussed by Gargett et al. (1981) in that the energy density is roughly independent of vertical wavenumber $(k)$ on vertical scales longer than $10 \mathrm{~m}$, and decays as $k^{-1}$ on smaller scales. The tilt-corrected spectra exhibit no anomalous features on scales cor-
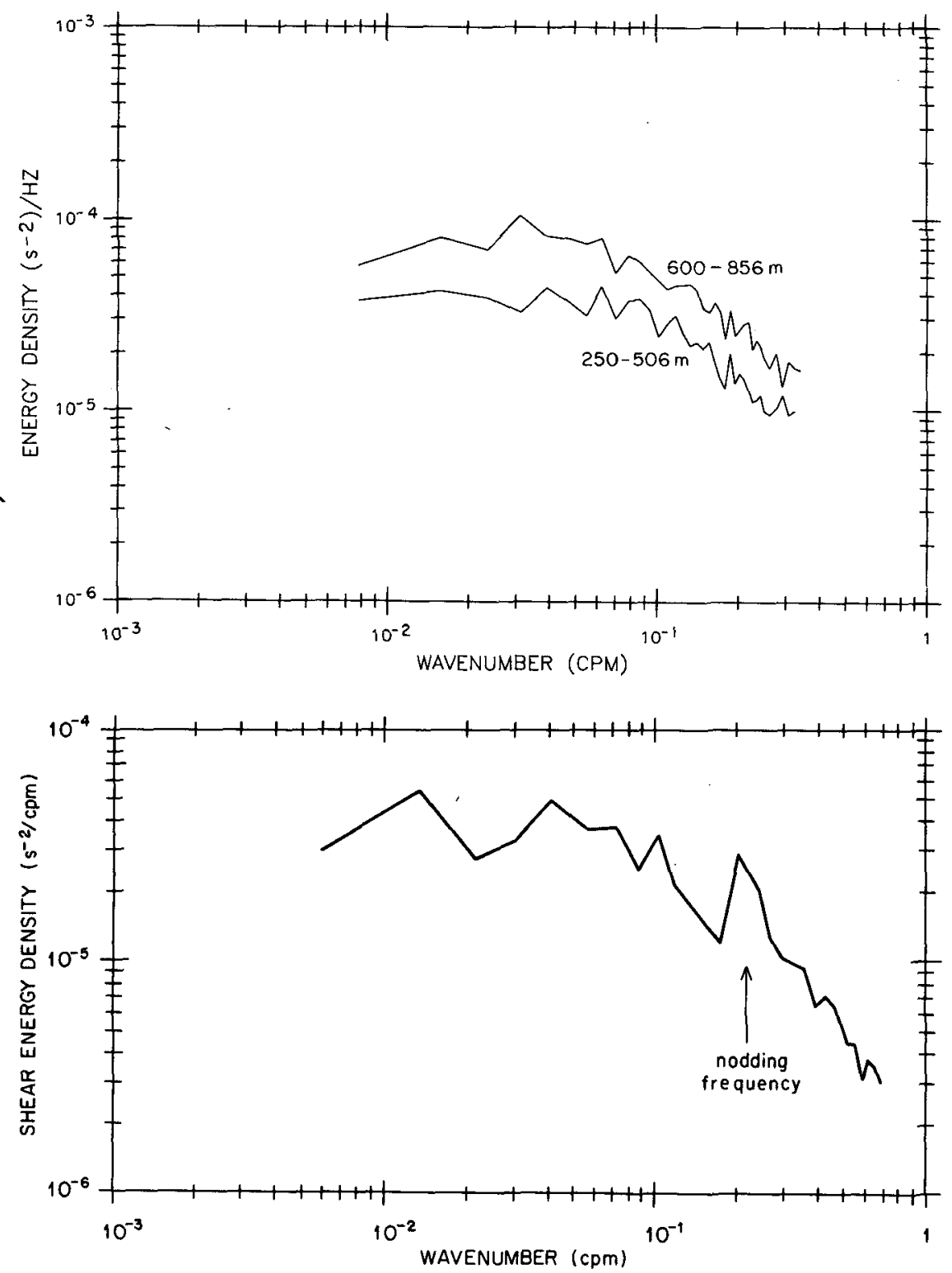

FIG. 10. (a) Ensembled averaged vertical wavenumber spectra of the vertical shear of horizontal currents. Data were obtained in the Sargasso Sea from the depth interval 250-506 m and 600$856 \mathrm{~m}$. Higher energy levels were observed in the deeper depth interval where the stratification was greater. The spectra are cut off at 0.3 cycles per meter because of noise in the acoustic current meter circuitry. (b) Spectrum of shear obtained from the point mass model without correction for instrument tilting. A pronounced peak in energy density is observed at scales corresponding to the instrument tilting frequency. 


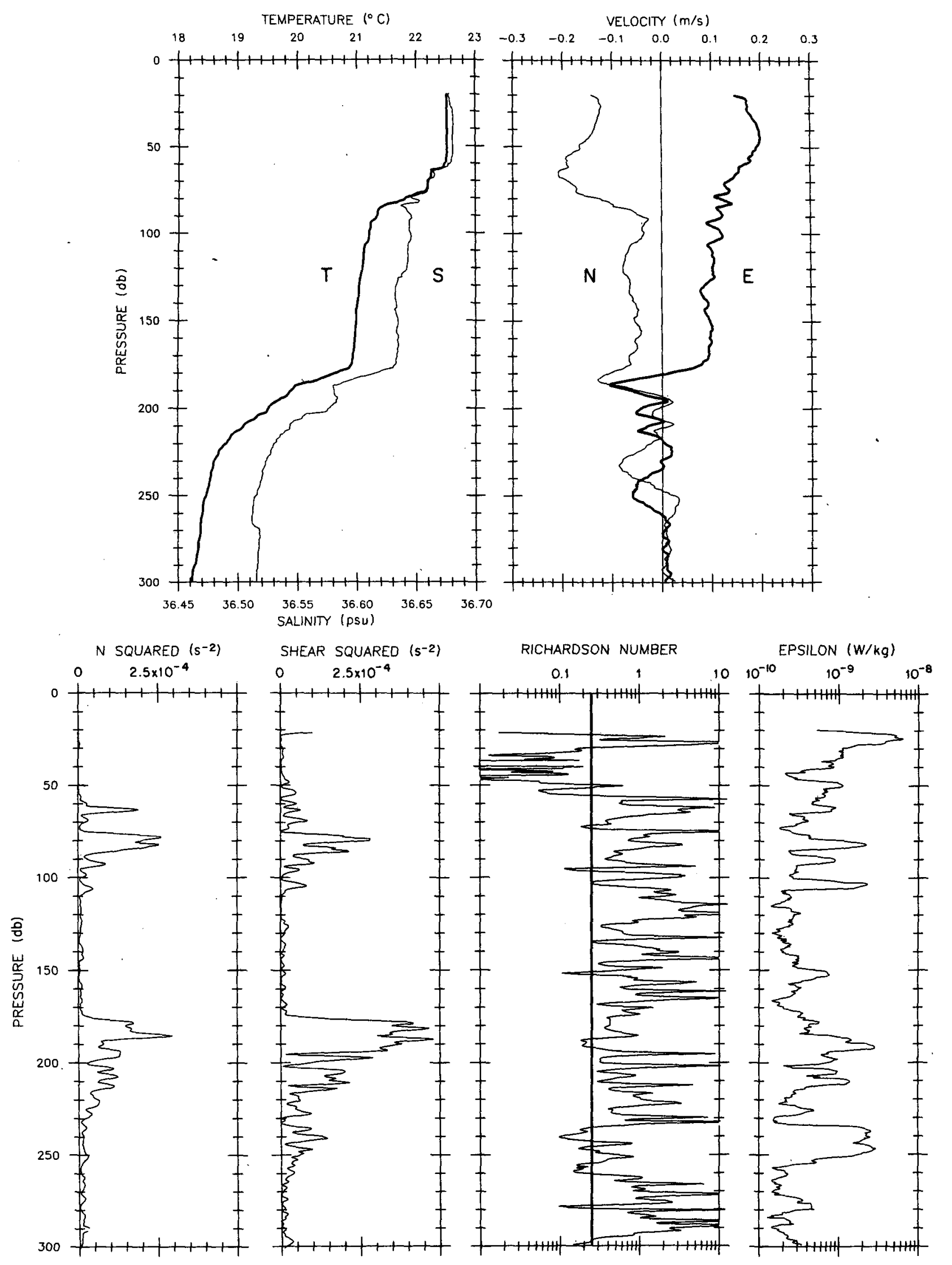

FIG. 11. (a) Vertical profiles of temperature, salinty and the two components of velocity obtained at the subtropical convergence front during FASINEX. The frontal velocities are associated with the two layer flow in the upper $200 \mathrm{~m}$. (b) Profiles of squared buoyancy frequency $\left(N^{2}\right)$, shear squared $\left(u_{z}{ }^{2}+v_{z}{ }^{2}\right)$, Richardson number, and kinetic energy dissipation rate $(\epsilon)$. Vertical gradients of $N^{2}$ and shear squared were estimated across $3 \mathrm{~m}$ vertical intervals. Estimates of $\epsilon$ were obtained over $0.5 \mathrm{~m}$ intervals (assuming the velocity fluctuations were isotropic) and subsequently smoothed over $3 \mathrm{~m}$. 
responding to the pendulum period of the instrument (about $0.2 \mathrm{cpm}$ ).

\section{Discussion and conclusions}

A new profiler has been developed for the study of ocean fine- and microstructure. It is capable of resolving the density and velocity variability of the ocean from full ocean depth to $1-\mathrm{cm}$ scales. It features: microprocessor control of operations, data acquisition and storage, solid state memory, a battery capacity sufficient for 20 to 40 dives, and a special handling rig which provides for ease of deployment and recovery. The great length of the instrument and its large moment of inertia mean that it has a better response function than earlier instruments for the measurement of the ocean velocities. In addition, the use of two pairs of accelerometers allows for the correction of the effect of instrument tilt on the velocity profile.

The instrument was used for the first time during the Frontal Air-Sea Interaction Experiment (FASINEX, Stage and Weller 1985) in February and March of 1986. The profiler worked in nearly flawless fashion, providing high quality data with a fairly simple operating routine. A crew of three is sufficient for at-sea operations; more personnel can be used if round-theclock operation is needed. The combination of fineand microscale sensors allows us to examine the physics of mixing processes in great detail (Toole and Schmitt 1987). Figure 11 is indicative of the capabilities of the new instrument. The velocity profile, (Fig. 1 la) taken near the Subtropical Convergence Front southwest of Bermuda, reveals a striking two-layer flow in the upper $200 \mathrm{~m}$, with the sharp gradient in the north component of velocity between 70 and $90 \mathrm{~m}$ depth coincident with the rapid change in temperature and salinity. The water properties of the thermostad between 90 and $190 \mathrm{~m}$ depth are identical to those in the surface layer on the cold side of the front, suggesting a relatively simple two layer frontal structure.

Smaller scale features are also prominent in the profile. High wavenumber velocity structures are seen at depths where the stratification is large, particularly at the base of the second thermostad. Local estimates of buoyancy frequency $\left(N^{2}\right)$ and shear squared, computed over $3 \mathrm{~m}$ intervals, Fig. $11 \mathrm{~b}$, exhibit similar behavior with depth. Correlation between $N^{2}$ and shear-squared is predicted by internal wave scaling arguments. Richardson number, also computed over $\mathbf{3} \mathrm{m}$ interval, attains low values at levels where small scale velocity features are found. Several occurrences of $R i \leqslant 1 / 4$ are found in the profile, but the vertical extent of each feature is small, of order $5 \mathrm{~m}$. The $\mathrm{Ri}$, estimated over $10-\mathrm{m}$ vertical scale, seldom attains values less than $1 / 4$, a finding which confirms the necessity for obtaining finescale information on vertical scales of order $1 \mathrm{~m}$. Kinetic energy dissipation rate was estimated on this profile as well. The profile plot suggests that elevated $\epsilon$ levels are more likely to occur at depths where $\mathrm{Ri}$ $\leqslant 1 / 4$, implicating shear instability as a prominent mixing mechanism at the FASINEX site. Analysis of the full FASINEX dataset is proceeding to quantify the mechanisms and rates of mixing near the front.

In summary, a new High Resolution Profiler is available for the study of ocean finestructure, microstructure and mixing. The combination of sensors permits investigation of the causative agents of mixing which should contribute significantly to our understanding of small scale ocean physics. Use of the profiler in a variety of oceanic environments, depths, and mixing regimes is being planned.

Acknowledgments. A great number of people helped in the design and fabrication of the instrument at WHOI. These include: K. Fairhurst, M. Woodward, K. Wannop and J. Dellibovi. T. Danforth, K. Prada and T. Sgouros helped to develop software. A. Martin assisted with the Micro-VAX computer. M. WoodgateJones assisted with data processing. Neil Oakey of Bedford Institute generously supplied advice on microstructure instrumentation and lent us shear probes during FASINEX. A. Williams and C. Converse developed the shadowgraph instrument and loaned us recovery aids for the cruise. S. Hayes of the Pacific Marine Environmental Laboratory provided advice and software. $R$. Lueck provided software and a sharp critique of the manuscript. We thank the Captain and crew of the R/V Endeavor for their role in our highly successful first cruise. The profiler was developed with funds from the Department of Defense Instrumentation program (Grant N00014-83-G-0161) and the Office of Naval Research (Contract N00014-82-C-0019, NR 083-004). The National Science Foundation supported the development of the shadowgraph instrument (Grant OCE84-9323) and provided ship time for the first use of the profiler (Grant OCE85-15336).

\section{REFERENCES}

Batchelor, G. K., 1959: Small-scale variation in convected quantities like temperature in a turbulent fluid. $J$. Fluid Mech., 5, 113133.

Bendat, J. S., and A. G. Piersol, 1971: Random Data: Analysis and Measurement Procedures. Wiley-Interscience, 231-232.

Caldwell, D. R., 1983: Small-scale physics in the ocean. Rev. Geophys. Space Phys., 21, 1192-1205.

Childers, D. G., and A. Durling, 1975: Digital Filtering and Signal Processing. West Publishing. $151 \mathrm{pp}$.

Converse, C. H., A. J. Williams, P. D. Fucille and R. W. Schmitt, 1986: A Free Ocean Vehicle to Measure Optical Microstructure. Current Practices and New Technology in Ocean Engineering, Vol. 11, 341-345.

Desaubies, Y., and W. K. Smith, 1982: Statistics of Richardson number and instability in oceanic internal waves. J. Phys. Oceanogr., 12(11), 1245-1259.

Dillon, T. M., 1982: Vertical overturns: A comparison of Thorpe and Ozmidov length scales. J. Geophys. Res., 87(C12), 96019613.

Evans, D. L., H. T. Rossby, M. Mork and T. Gytre, 1979: YVETTE, A free-fall shear profiler. Deep-Sea Res., 26, 703-718.

Gargett, A. E., and R. W. Schmitt, 1982: Observations of salt fingers 
in the central waters of the eastern North Pacific. J. Geophys. Res., 87(C10), 8017-8029.

_- P. J. Hendricks, T. B. Sanford, T. R. Osborn and A. J. Williams, 3rd., 1981: A composite spectrum of vertical shear in the upper ocean. J. Phys. Oceanogr., 11, 1258-1271.

Georgi, D. T., R. C. Millard and R. W. Schmitt, 1983: Conductivity microstructure measurements with a CTD. IEEE Proc. Third Working Symp. on Oceanographic Data Systems. 5-13 pp.

Gregg, M. C., 1979: The effects of bias error and system noise on parameters computed from C, T, P and V profiles. J. Phys. Oceanogr., 9(1), 199-217.

thermistors. J. Geophys. Res., 85, 2779-2786.

$\longrightarrow,-$ A. Pederson and E. Aagaard, 1978: Low noise temperature microstructure measurements with thermistors. Deep-Sea Res., 25, 843-856.

—- E. A. D'Asaro, T. J. Shay and N. Larson, 1986: Observations of persistent mixing and near-inertial internal waves. $J$. Phys. Oceangr., 16(5), 856-885.

Hayes, S. P., H. B. Milburn and E. F. Ford, 1984: TOPS: A free fall velocity and CTD profiler. J. Atmos. Oceanic Technol. 1(3), 220-236.

Horne, E. P. W., and J. M. Toole, 1980: Sensor response mismatches and lag correction techniques for temperature-salinity profilers. J. Phys. Oceanogr., 10(7), 1122-1130.

Kunze, E., A. J. Williams, III and R. W. Schmitt, 1987: Optical microstructure in the thermohaline staircase east of Barbados. Deep-Sea Res., 34, 1697-1704.

Linden, P. F., 1974: Salt fingers in a steady shear flow. Geophys. Fluid Dyn., 6, 1-27.

Lueck, R. G., and T. R. Osborn, 1980: The characteristics of internally heated thermistors. Deep-Sea Res., 27, 273-292.

-, O. Hertzman and T. R. Osborn, 1977: The spectral response of thermistors. Deep-Sea Res., 24, 951-970.

Luyten, J. R., G. Needell and J. Thomson, 1982: An acoustic dropsonde-the White Horse: Design, performance and evaluation. Deep-Sea Res., 29, 499-524.

Magnell, G., 1976: Salt fingers observed in the Mediterranean outflow region $\left(34^{\circ} \mathrm{N}, 11^{\circ} \mathrm{W}\right)$ using a towed sensor. J. Phys. Oceanogr., $6,511-523$.

Mellinger, E. 1986: High density static RAM for in-situ bulk data storage. Proc. IEEE Oceans '86, 444-447.

—, K. E. Prada, R. L. Koehler and K. W. Doherty, 1986: Instrument Bus: An Electronic System Architecture for Oceanographic Instrumentation. Woods Hole Oceanogr. Inst. Tech. Rep., WHOI-86-30, $86 \mathrm{pp}$.
Meagher, T., A. Pedersen and M. Gregg, 1982: A low noise conductivity, microstructure instrument. Proc. of IEEE Oceans '82, 283-290.

Millard, R. C., and N. Galbraith, 1982: WHOI Processed CTD Data Organization. Woods Hole Oceanogr. Inst. Tech. Rep., WHOI$82-37,36 \mathrm{pp}$.

Oakey, N. A., 1977: An Instrument to Measure Oceanic Turbulence and Microstructure. Bedford Inst. Oceanogr. Rep. Ser. BI-R$77-3,52 \mathrm{pp}$.

- 1982: Determination of the rate of dissipation of turbulent energy from simultaneous temperature and velocity microstructure measurement. J. Phys. Oceanogr., 12, 256-271.

Osborn, T. R., 1974: Vertical profiling of velocity microstructure. Journal Phys. Oceanogr., 4, 109-115.

- , and W. R. Crawford, 1980: An airfoil probe for measuring turbulent veloicity fluctuations in water. Air-Sea Interactions: Instruments and Methods, F. Dobson, L. Hasse, and R. Davis, Eds., Plenum Press, 801 pp.

—_, and C. S. Cox, 1980. Oceanic finestructure. Geophys. Fluid Dyn., 3, 321-345.

Schmitt, R. W., 1981: Form of the temperature-salinity relationship in the Central Water: Evidence for double-diffuse mixing. $J$. Phys. Oceanogr., 11(7), 1015-1026.

- 1987: The Caribbean sheets and layers (C-SALT) Program. Eos, 68(5), 57-60.

- , and D. L. Evans, 1978: An estimate of the vertical mixing due to salt fingers based on observations in the North Atlantic Central Water. J. Geophys. Res., 83, 2913-2919.

- , and D. T. Georgi, 1982: Finestructure and microstructure in the North Atlantic Current. J. Mar. Res., 40(Suppl.), 659-705.

Stage, S. A., and R. A. Weller, 1985: The Frontal Air-Sea Interaction Experiment (FASINEX); Part 1: Background and scientific objectives. Bull. Am. Meteor. Soc., 66(12), 1511-1520.

Toole, J. M., and R. W. Schmitt, 1987: Fine- and microscale structures in the NW Atlantic subtropical front. Nature, 327(6117), 4749.

__ and S. P. Hayes, 1984: Finescale velocity-density characteristics and Richardson number statistics of the eastern equatorial Pacific. J. Phys. Oceanogr. 14(4), 712-726.

Vachon, P., and R. G. Lueck, 1984: A small combined temperatureconductivity probe. Proc. 1984 STD Conf. and Workshop. Mar.Tech. Soc. and MTS Oceanic Instrumentation Committee, $126-131$.

Williams, A. J., 1975: Images of ocean microstructure. Deep-Sea Res., 22, 811-829. 